\title{
Non-Hodgkin's Lymphoma of Thyroid Synchronously with Squamous Cell Carcinoma Base of Tongue: A Rare Coincidence and Treatment Strategy
}

\author{
${ }_{1}^{1}$ Paramjeet Kaur, ${ }^{2}$ Anil Khurana, ${ }^{3}$ Ashok K Chauhan, ${ }^{4}$ Gajender Singh, ${ }^{5}$ Sant Parkash Kataria
}

\begin{abstract}
The occurrence of two malignant lesions synchronously of different histology at same anatomical region of the patient is a rare presentation. Coexistence of squamous cell carcinoma and malignant lymphoma of head and neck region synchronously is uncommon. Here is a report of case of 65 years old female patient who presented with a mass in right side of neck and in midline of neck of 2 months duration. After thorough work up, the patient was diagnosed as having squamous cell carcinoma of right side base of tongue and non-Hodgkin's lymphoma $(\mathrm{NHL})$ of thyroid. The patient was treated with radiotherapy and chemotherapy.
\end{abstract}

Keywords: Thyroid, Non-Hodgkin's lymphoma, Base of tongue squamous cell carcinoma.

How to cite this article: Kaur P, Khurana A, Chauhan AK, Singh G, Kataria SP. Non-Hodgkin's Lymphoma of Thyroid Synchronously with Squamous Cell Carcinoma Base of Tongue: A Rare Coincidence and Treatment Strategy. Int J Head Neck Surg 2014;5(3):155-157.

Source of support: Nil

Conflict of interest: None

\section{INTRODUCTION}

Multiple primary neoplasms are described as two or more abnormal growth of tissue occurring simultaneously and presumed to be of separate origin. Synchronous lesions are those in which both lesions appear at the same time or within 2 months. ${ }^{1,2}$ Primary non-Hodgkin's lymphoma (NHL) of thyroid is an uncommon malignancy. The second malignancy in this case was squamous cell carcinoma of base of tongue; both are presumed to be

\footnotetext{
${ }^{1,4}$ Associate Professor, ${ }^{2}$ Senior Resident

${ }^{3}$ Senior Professor, ${ }^{5}$ Professor

${ }^{1-3}$ Department of Radiotherapy, Pandit Bhagwat Dayal Sharma Post Graduate Institute of Medical Sciences, Rohtak, Haryana India

${ }^{4,5}$ Department of Pathology, Pandit Bhagwat Dayal Sharma Post Graduate Institute of Medical Sciences, Rohtak, Haryana India
}

Corresponding Author: Paramjeet Kaur, Associate Professor Department of Radiotherapy, Pandit Bhagwat Dayal Sharma Post Graduate Institute of Medical Sciences, Rohtak, Haryana India, Phone: 01262210591, e-mail: drparamjitkaur@ rediffmail.com having different etiology, natural history, histopathology, treatment strategies and outcome.

The cases of double malignancies may cause the problem of optimal treatment for the patient. Treatment strategies in case of synchronous malignancies depend on treating the malignancy that is more advanced first or sometimes both could be treated simultaneously. The case presented here is illustrating the dilemma involved in clinical decision making on treatment in a patient with two primaries with dissimilar histology in same anatomical region.

\section{CASE REPORT}

A 65 years old postmenopausal female presented with swelling in midline of neck of 2 months duration. There was pain on right side of neck and, dyspnea, hoarseness and difficulty in swallowing for solids food. On examination, there was $5 \times 5 \mathrm{~cm}$ swelling in midline of neck firm, tender that moves with deglutition (Fig. 1). Bilateral multiple cervical lymph nodes were palpable. No other lymph nodes were palpable.

On oral cavity, tongue mobility and mouth opening was normal. A large growth was palpable over right side base of tongue, extending toward tonsillolingual sulcus. Contrast-enhanced computed tomography (CECT) neck showed heterogeneously enhancing hypodense lesion with low-density area in base of tongue mainly on right side, crossing midline. Enlarged nodes were seen in

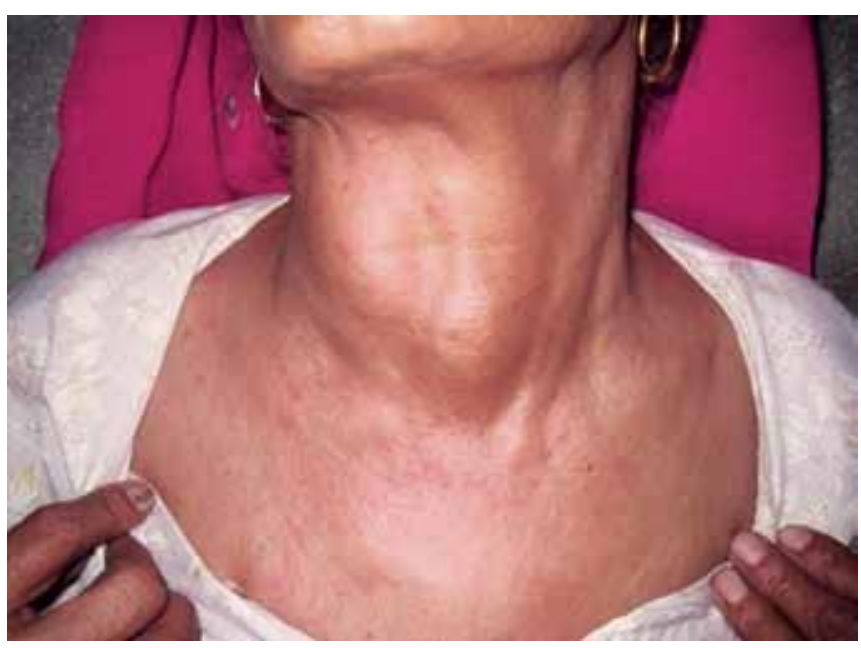

Fig. 1: Swelling midline neck region 


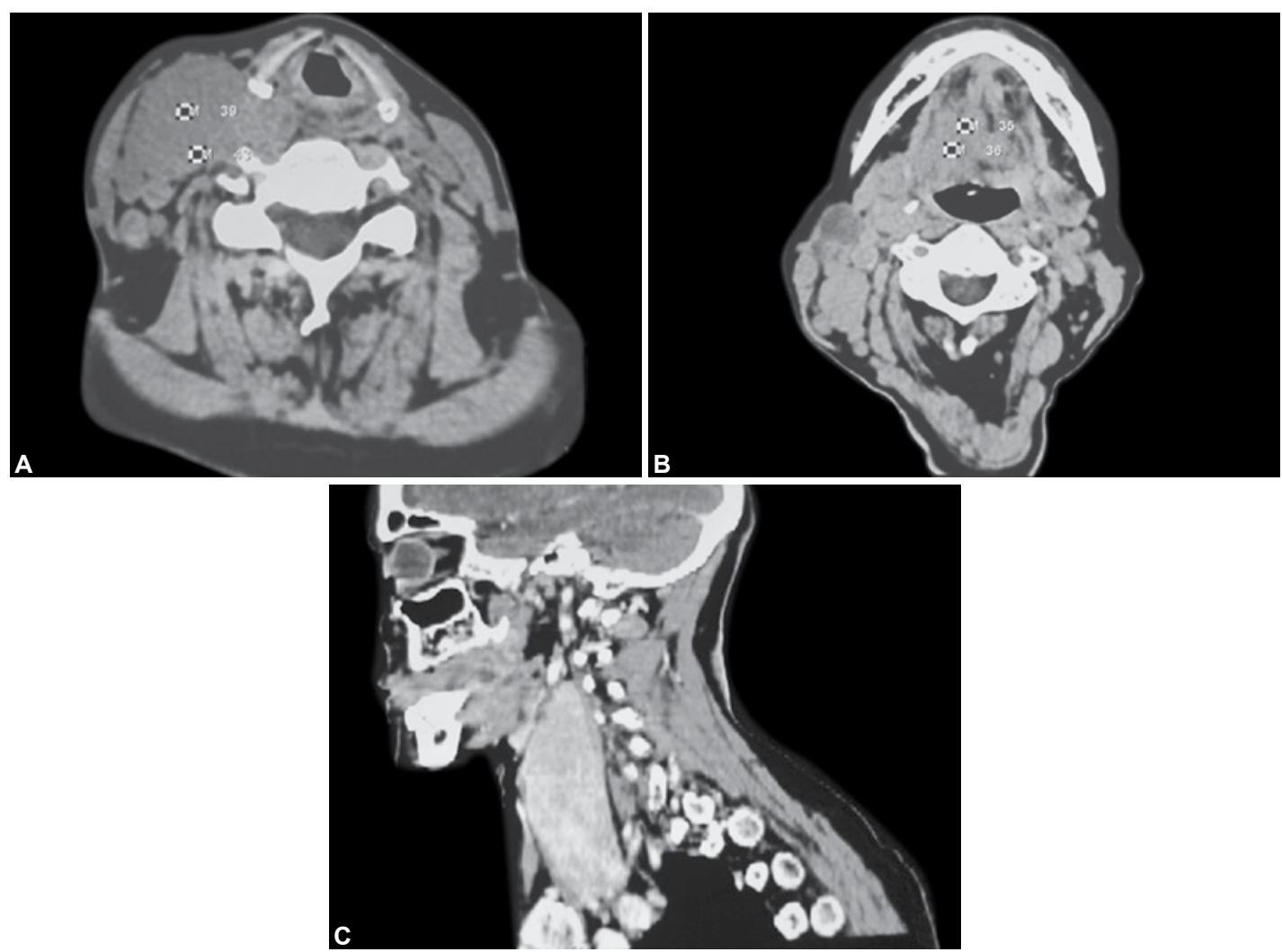

Figs 2A to C: Contrast enhanced computed tomography scan of face and neck showing growth at base of tongue, cervical lymph nodes and thyroid

bilateral cervical chain more in left side with necrosis in few nodes. Bilateral lobes and isthmus of thyroid were enlarged and altered in size and shape with hypodensities (Figs 2A to C).

Histopathological examination of growth base of tongue and thyroid showed moderately differentiated squamous cell carcinoma and diffuse large cell B cell NHL respectively (Fig. 3). Immunohistochemisty examination of thyroid was positive for leukocytes common antigen and negative for cytokeratin. Fine needle aspiration cytology of cervical lymphnodes also revealed metastases from squamous cell carcinoma. After confirmation of diagnosis NHL thyroid, the patient was investigated to rule out extrathyroidal extension. Thyroid-stimulating hormone (TSH) was $2.2 \mathrm{U} / \mathrm{L}$.

The patient was finally diagnosed having double malignancy synchronously, moderately differentiated squamous cell carcinoma base of tongue (staged $\mathrm{T}_{3} \mathrm{~N}_{2 \mathrm{c}} \mathrm{M}_{0}$ ) and primary NHL of thyroid (stage IE). The patient was given radical radiotherapy. The dose of radiation prescribed was 44 Gy in 22 fractions over 4 weeks, by anterior posterior and posterior anterior field to lower face and neck extending $2 \mathrm{~cm}$ below the infraclavicular fossae. The patient was given boost $20 \mathrm{~Gy}$ by parallel opposed fields to base of tongue while sparing spinal cord and thyroid. At 3 months follow-up, base of tongue and thyroid swelling showed complete regression of disease, but nodal sites showed partial response. Modified neck dissection performed, the histopathological examination revealed metastases from squamous cell carcinoma. Then, the patient received combination chemotherapy with paclitaxel $(260 \mathrm{mg})$ and carboplatin $(450 \mathrm{mg})$ three weekly for six cycles. The patient is asymptomatic with no evidence of disease at both primary sites after 8 months of completion of treatment.

\section{DISCUSSION}

The incidence of multiple primary cancers is rare and reported to be between 0.3 and $4.3 \%$. The phenomenon

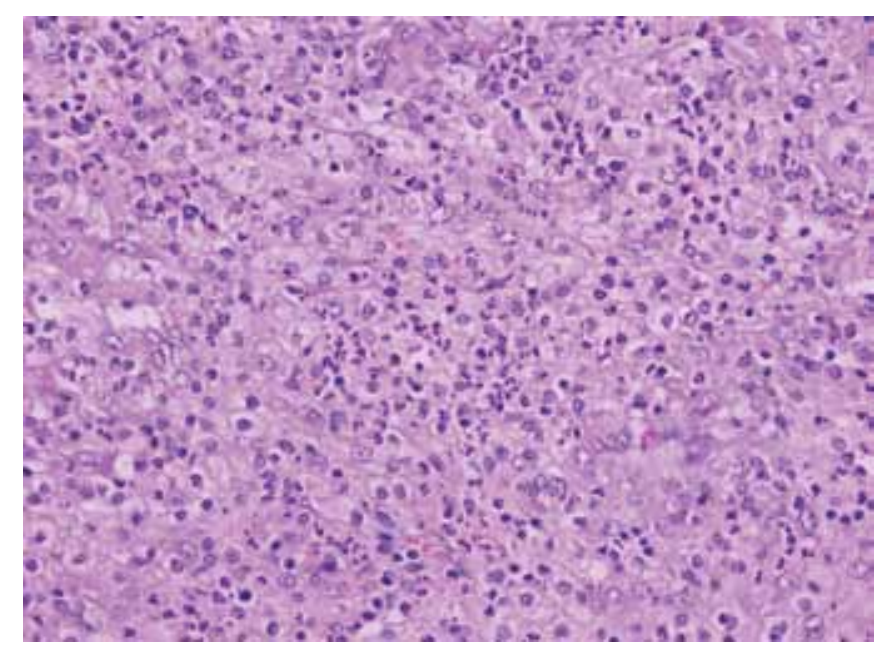

Fig. 3: Photomicrograph showing large lymphoid cells with high $\mathrm{N} / \mathrm{C}$ ratio and prominent nucleolus replacing normal thyroid gland architecture. Non-Hodgkin's lymphoma thyroid (H\&E: 400x) 
of multiple primary malignant neoplasms in the same individual was described firstly by Billroth. ${ }^{3}$

Non-Hodgkin's lymphoma of thyroid is uncommon and accounting for less than $2 \%$ of thyroid malignancies and 1 to $2 \%$ of extranodal lymphomas. ${ }^{4}$ Diffuse large B-cell lymphoma, mucosa-associated lymphoid tissue (MALT) lymphoma and mixed type are subtypes of thyroid NHL. Primary diffuse large B-cell lymphoma is commonest type (70\%) and MALT lymphomas comprise of 6 to $26 \%$ and have indolent course.

Current treatment modalities for thyroid lymphomas are radiotherapy and chemotherapy and surgical intervention is limited to diagnostic biopsy. Chemotherapy with cyclophosphamide, adriamycin, vincristine intravenous and oral prednisolone (CHOP regimen) followed by involved field radiation therapy has more frequently used for primary thyroid lymphoma., ${ }^{5,6}$ Diffuse large B cell variant of thyroid lymphomas have aggressive clinical course and over all 5 years survival is less than $50 \%{ }^{7}$

Radiation therapy with or without chemotherapy is mainstay of treatment for locally advanced carcinoma of oropharynx. With definitive radiotherapy 5 years local control observed in T3 stage $82 \%$ and for stage IVA 5 years local control rate, absolute and cause specific survival observed 87,67 and $84 \%$ respectively. ${ }^{8}$

Therefore, radiation therapy was used as initial treatment modality in present case and both primaries were treated in single field at same time. Since this patient showed a complete response at primary sites but response at nodal sites was suboptimal; therefore, she underwent modified neck dissection.

The chemotherapy is employed for carcinoma base of tongue in view of better 5 years survival in contrast to diffuse large B cell lymphoma thyroid (67 vs 50\%). Moreover, neck dissection offers significant improved local control and disease-free survival.

Watanabe et al reported a case of double malignancies including oropharyngeal squamous cell carcinoma and nasopharyngeal lymphoma and recommended that chemotherapy should be administered for the disease with the higher-survival rate after the disease with lowsurvival rate showed complete remission. ${ }^{9}$

In present case, adjuvant chemotherapy with paclitaxel and carboplatin was administered in patient three weekly for six cycles.

Eight months after the treatment, the patient was asymptomatic and with no evidence of disease at both sites.
Improving and better understanding of biological behavior of disease and subtype of tumor is clinically relevant to assess clinical course, outcome and the choice of therapy in case of synchronous malignancies. However, in comparison to metachronous malignancies, the prognosis of synchronous tumors is significantly lower. Di Martino et al in a study with two malignancies, an aggressive treatment strategy was employed yielded the most favorable results, with a 5 years survival rate of 66.8 and $35.9 \%$ for index tumors and second primary malignancies respectively. ${ }^{2}$

\section{CONCLUSION}

In present case, both malignancies were in same anatomical region, therefore, treated simultaneously with radiation therapy. Patients with synchronous malignancies should be treated at same time if possible depending upon biological behavior and clinical course.

\section{REFERENCES}

1. Howe HL. A review of the definition for multiple primary cancers in the United States. Workshop proceedings from December 4-6, 2002, in Princeton. North American Association of Central Cancer Registries: New Jersey: Springfield (IL); 2003 May.

2. Di Martino E, Sellhaus B, Hausmann R, et al. Survival in second primary malignancies of patients with head and neck cancer. J Laryngol Otol 2002 Oct;116(10):831-838.

3. Billroth T. Die agemeine chirurgische pathologie und therapie. Reimer G, editor, 14 Aufl Berlin; 1889. p. 908.

4. Graff-baker A, Roman SA, Thomas DC, et al. Prognosis of primary thyroid lymphoma: demographics, clinical and pathologic predictors of survival in 1408 cases. Surgery 2009; 146(6):1105-1115.

5. Coiffier B, Lepage E, Briere J, et al. CHOP chemotherapy plus rituximab compared with $\mathrm{CHOP}$ alone in elderly patients with diffuse large B cell lymphoma. N Engl J Med 2002 Jan;346(4):235-242.

6. Sehn LH, Donaldson J, Chhanabhai M, et al. Introduction of combined $\mathrm{CHOP}$ plus ritiuximab therapy dramatically improved outcome of diffuse large B-cell lymphoma in British Columbia. J Clin Oncol 2005 Aug;23(22):5027-5033.

7. Widder S, Pasieka JL. Primary thyroid lymphoma. Curr Treat Options Oncol 20042004 Aug;5(4):307-313.

8. Mendenhall WM, Morris CG, Amdur RJ, Hierman RW, Werning JW, Villaret DB. Definitive radiotherapy for squamous cell carcinoma of the base of tongue. Am J Clin Oncol 2006;29(1):32-39.

9. Watanabe N, Inohara H, Akahani S, Yamamoto Y, Moriwaki K, Kubo T. Synchronous squamous cell carcinoma and malignant lymphoma in head and neck region. Auris Nasus Larynx 2007;34(2):273-276. 